Molecular Weight Distributions of Irradiated Siloxane-Based Elastomers: A Complementary Study by Statistical Modeling and Multiple Quantum Nuclear Magnetic Resonance.

L. N. Dinh, B. P. Mayer, A. Maiti, S. C. Chinn, R. S. Maxwell

December 8, 2010

Journal of Applied Physics 
This document was prepared as an account of work sponsored by an agency of the United States government. Neither the United States government nor Lawrence Livermore National Security, LLC, nor any of their employees makes any warranty, expressed or implied, or assumes any legal liability or responsibility for the accuracy, completeness, or usefulness of any information, apparatus, product, or process disclosed, or represents that its use would not infringe privately owned rights. Reference herein to any specific commercial product, process, or service by trade name, trademark, manufacturer, or otherwise does not necessarily constitute or imply its endorsement, recommendation, or favoring by the United States government or Lawrence Livermore National Security, LLC. The views and opinions of authors expressed herein do not necessarily state or reflect those of the United States government or Lawrence Livermore National Security, LLC, and shall not be used for advertising or product endorsement purposes. 


\title{
Molecular Weight Distributions of Irradiated Siloxane-Based Elastomers: A Complementary Study by Statistical Modeling and Multiple Quantum Nuclear Magnetic Resonance.
}

\author{
L. N. Dinh, ${ }^{*}$ B. P. Mayer, A. Maiti, S. C. Chinn, R. S. Maxwell \\ Lawrence Livermore National Laboratory
}

\section{ABSTRACT:}

The statistical methodology of population balance $(\mathrm{PB})$ has been applied to predict the effects of cross-linking and chain-scissioning induced by ionizing radiation on the distribution of molecular weight between cross-links (MWBC) of a siloxane-based elastomer. Effective molecular weight distributions were extracted from the quantification of residual dipolar couplings by multiple quantum nuclear magnetic resonance (MQ-NMR) measurements and are taken to reflect actual MWBC distributions. The PB methodology is then applied to the unirradiated MWBC distribution and considers both chain-scissioning and the possibility of formation of three types of cross-links: random recombination of scissioned-chain ends (endlinking), random covalent bonds of free radicals on scissioned-chain ends (Y-cross-linking), and formation of random cross-links from free radicals on side groups (H-cross-linking). The qualitative agreement between the statistical modeling approach and NMR data confirms that it is possible to predict trends for the evolution of the distribution of MWBC of polymers under irradiation. The approach described herein can also discern heterogeneities in radiation effects in different structural motifs in the polymer network. 
*Author to whom correspondence should be sent:

Dr. L. N. Dinh

Lawrence Livermore National Laboratory

P. O. Box 808, Mail Stop: L-091, Livermore, CA 94551

Phone: (925) 422-4271

Fax: $\quad(925) 423-8772$

E-mail: dinh1@1lnl.gov 


\section{INTRODUCTION:}

Silica-filled polydimethylsiloxane (PDMS) composite cross-linked materials, broadly referred to as silicones, are of great appeal due to their environmental resilience, biocompatibility, broad technological applications, and their availability in a wide range of tailor-made chemical and mechanical properties [1-3]. Unfortunately, many siloxane-based compounds undergo degradation such as outgassing and stiffening with time in a dry environment [4], or modification of polymer chain lengths by exposure to radiation or elevated temperatures [5-13]. The changes to the network structure are understood to occur by competing chain-scissioning and cross-linking reactions $[6,13]$. The relative contributions of these competing reactions can be a function of polymer sequence, the chain length (or mobility), the temperature, and the type of incident radiation. Because these dependencies may be interconnected or non-linear with dose or time, their effects are thus generally difficult to predict $[6,14-15]$.

A number of studies on the degradation of siloxane polymers by irradiation concentrate on the calculation of the relative ratios of cross-linking to chain-scissioning, either via the use of Charlesby-Pinner analyses of the sol-fraction [16] or via a statistical approach first outlined by Saito [17]. The most satisfying practice is probably the analysis of molecular weight distributions obtained by gel permeation chromatography (GPC) from irradiated soluble polymer chains [17-19]. For cross-linked networks, however, the use of GPC to obtain insight into the effects of radiation on the distributions of the chain lengths in the polymer network is not feasible since network chains are insoluble. The Charlesby-Pinner method provides some help in alleviating this limitation by extracting details about the gel fraction indirectly through investigation of the dose-dependent evolution of the soluble polymer fraction. However, the 
ability of this method to provide robust, long-term predictions is limited since the size of this soluble fraction is generally only on the order of a few percent, and measurement uncertainty must be low in order to derive meaningful conclusions.

Multiple quantum nuclear m agnetic resonance (MQ-NMR) has been used with much success over the past decade to reveal detailed information on polymer dynamics, microstructure, and degradation [20-27]. In $m$ any of these studies, relative changes in polym eric properties are tracked in considerable detail, a llowing for the extraction and the id entification of otherwise subtle or invisible chem ical and physical pheno mena. MQ-NMR is sensitive to a variety of topological constraints that define typical siloxane network stru ctures, but detailed analyses which aim to parameterize these structures is lacking. The goals of the present work, then, are two-fold: 1) the analys is of the changes in $\mathrm{m}$ olecular weight distributions derived from MQNMR residual dipolar coupling measurements, and 2) the appl ication of the statistical methodology of population balance to predict the e volution of the molecular weight distribution as a function of cumulative gamma irradiatio $\mathrm{n}$ dosage using solely MQ-NMR data for the unirradiated material.

\section{MQ-NMR DERIVED SILOXANE NETWORK STRUCTURE:}

MQ-NMR allows for the quantification of dipolar couplings between protons on polymer chains experiencing a variety of topological constr aints, both chem ical and physical in nature, which prevent the complete motional averaging of the homonuclear $\left({ }^{1} \mathrm{H}-{ }^{1} \mathrm{H}\right)$ dipolar interaction. The parameter that quantifies th e resultant scaling through chain segm ental dynamics of the nominal dipolar coupling strength is referred to as the residual dipolar coupling magnitude. The relationship between this residual dipolar coupling (RDC), $D_{\text {res }}$, and the num ber of effective statistical segments between two constraints, $N_{c}$, is given by: 
$\frac{D_{\text {res }}}{D_{\text {stat }}}=\frac{3}{5} \frac{r^{2}}{N_{c}}\left\langle P_{2}(\cos \alpha)\right\rangle$

where $r^{2}$ (assumed herein to be unity) is the squ ared end-to-end vector length norm alized by its unperturbed, melt state value; $D_{\text {stat }}$ is the static dipolar coupling value $(8.9 \mathrm{kHz}$ when considering rapidly rotating methyl groups); and $\left\langle P_{2}(\cos \alpha)\right\rangle$ is the tim e-averaged second order Legendre polynomial of the cosine of $\alpha$, the angle between the $\mathrm{Si}-\mathrm{C}$ vector and the chain axis. This term takes into account rapid, intras egmental motions (isomerizations) that im part cylindrical-type symmetry to a given segm ent. Assuming this angle $\alpha$ is roughly $90^{\circ}$, the time average value is therefore taken to be 2 (assum ing backbone rotational ergodicity). The prefactor of $3 / 5$ arises from considering average segmental orientations over the end-to-end vector of an idealized chain [28]. Ultim ately, Eqn. (1) can be thought of as expressing the an isotropy of the dipo lar interaction dictated by the correlation between semi-local segmental motion and the chain endto-end vector.

As discussed in detail previously, distribu tions of RDCs can be extracted for polymeric materials by probing multiple quantum coherences among protons along the chain [22-27]. In these works, a "bu ild-up" of thes e multiple quantum (MQ) coherences is measured through a suitable NMR experim ent, and the RDC distrib ution is typically ex tracted from this data in a model-free way using T ikhonov regularization software, FTIKREG [20]. This program is able to derive a series of possible normalized RDC distributions that would $\mathrm{m}$ anifest the observed NMR response; and through a detail ed statistical treatment of many such distributions, a "m ost likely" candidate can be chosen [27].

Eqn. (1) can be subsequently employed to convert the spectrum of residual dipolar couplings, as determined by the re gularization package, into an ef fective molecular weight distribution. $N_{c}$ is converted to an effective chain length, $p$, by simply multiplying it by the 
characteristic ratio, $C_{\infty}$, of PDMS (assumed here to be in the large molecular weight limit) of 6.43 $[21,29]$. Though it is stri ctly incorrect to say $C_{\infty}$ represents the number of backbone bonds per segment $N_{c}$, this approximation holds reasonably well for polymers with large valence angles, as is the case for PDMS. And though only the degree of polymerization herein is considered, $p$ can be easily converted into a molecular weight but multiplying by $74 \mathrm{~g} / \mathrm{mol}$, the nom inal PDMS monomer molecular weight. Ultim ately, the determination of the ef fective degree of polymerization, $p$, can be mathematically expressed by:

$$
p=\frac{3}{5} \frac{D_{\text {stat }}}{D_{\text {res }}} \frac{1}{P_{2}(\cos \alpha)} C_{\infty}=\frac{3}{10} \frac{D_{\text {stat }}}{D_{\text {res }}} C_{\infty}=\frac{17.2 \mathrm{kHz}}{D_{\text {res }}}
$$

In Eqn. (2), the right-most expression is roughly consistent with previous work [30].

Concerning the distributions of proton populations, special care must be taken to ensure that the relative areas (i.e., popul ation data) under the converted curve remain consistent, as direct application of Eqn. (1) fails to preserve area particularly due to the distorting effect of an inverse dependence of $N_{c}$ on $D_{\text {res. }}$. For example, small but non-zero populations around $D_{\text {res }}=0$ would lead to large, nonphysical populations of chains with an infinite effective $\mathrm{m}$ olecular weight if left uncorrected. This subtle, but crucial issue $\mathrm{c}$ an be addressed by using a sim ple spreadsheet-based numerical correction as was done for the present work. This protocol essentially amounts to converting $D_{\text {res }}$ data to $p$ (or molecular weight), then scaling the y-axis values such that the area between two consecutive $p$ values equals the area between their $D_{\text {res }}$ counterparts.

As reported earlier, the sensitivity of the MQ methodology is typically limited to molecular weights (MW) under the critical en tanglement MW, $M_{e}$, due to NMR's inability to discriminate between chemical and physical constraints in the present case [22]. The caveats and limitations of MQ-NMR-derived distributions have been discussed in detail elsewhere [28, 30] 
and will not be repeated here, except to note that the uncertainly associated with distributions for $p$ greater than 200 (roughly the degree of polym erization at $M_{e}$ ) and less than 10 is relativ ely large. This issue results from a combination of difficulties, including fitting faithfully MQ-NMR data in the plateau region (i.e., long MQ excitation times) and the inability to sufficiently sample at short excitation tim es. Because long and sho rt excitation times are sensitive to dynamics of long and short chain segm ents, respectively, gr eater uncertainty in the derived effective molecular weight distributions for these $p$ ranges is expected. Despite these and other limitations of the method and the assumptions required to develop Eqn. (1) and Eqn. (2), significant insights into the relative changes of polymer material properties due to chemical degradation have been previously reported [23, 25-27].

The molecular weight distributions obtained by applying Eqns. (1) and (2) to MQ-NMR data of TR55 (70 wt. \% polysiloxane gum formulation and $30 \mathrm{wt} . \%$ fumed silica filler pretreated with a silating agent) exposed to $0 \mathrm{kG} \quad \mathrm{y}, 10 \mathrm{kGy}, 50 \mathrm{kGy}, 100 \mathrm{kGy}$, and $250 \mathrm{kGy}$ at room temperature are shown in Fig. 1 on a sem i-log plot (a) and on a linear plot (b). The inset of Fig. 1(b) features the MWBC distri bution for $\mathrm{p}<30$. The symbol " $r$ " on the $\mathrm{y}$-axis in the figure represents the relative probability that a polymer has an ef fective chain length $p$ at a given radiation dose. It is im portant to emphasize th at the entire PB analysis as $\mathrm{d}$ iscussed below is derived from the NMR data in Fig. 1(b) alone.

\section{STATISTICAL DESCRIPTION OF CROSS-LINKING AND CHAIN-SCISSIONING OF POLYMERS IN A NETWORK:}

The goal of the PB methodology is the derivation of equations that describe the evolution of chain molecular weight as experimentally determined by NMR. The only key assumption is 
that cross-linking and chain-scissioning under irradiation is a random process (all monomer units are equally likely to undergo a positive event). A schematic depicting irradiation induced active center generation and chain-scissioning events are shown in Fig. 2. Active centers are formed through either broken $\mathrm{C}-\mathrm{H}$ or $\mathrm{Si}-\mathrm{C}$ bonds, while chain-scissioning involves a broken $\mathrm{Si}-\mathrm{O}$ bond. If the rate of change of the population of molecules with a degree of polymerization of $p$ in between any two restraints is of interest, it is sufficient to write down the rate of decrease in the population due to successful events within that same population and the rate of increase in the population due to contributions from events outside of that population. In the following discussion, the term "chains of length $p$ " refers to "chain length of $p$ between constraints".

\section{Cross-linking:}

If $D, c$, and $n(p, D)$ stand for the radiation dose, the rate of active center generation per unit radiation dose per monomer, and the number of chains of length $p$, respectively; then the decrease in the $p$ population due to the development of an active center within that population for an incremental dose, $\Delta D$, is: $p n(p, D) c \Delta D$ molecules. The probability of any chain developing two active centers is proportional to $(c \Delta D)^{2}$ and is negligibly small. Chains of length $p$ with an active center will cross-link with other molecules yielding shorter molecules in between crosslinks, accounting for the decrease in that population. Furthermore, molecules of non- $p$ chain length (called $l$ ) can also develop an active center, then cross-link with molecules within the $p$ population. This also acts to reduce the population of chains with length $p$. If $N$ is the total number of monomers in the sample, then this additional reduction in the population of length $p$ can be written as: $\frac{p n(p, D)}{N} \sum_{l=1}^{P_{\max }} \ln (l, D) c \Delta D=p n(p, D) c \Delta D$ chains. Thus, the total 
decrease in the population with the degree of polymerization of $p$ in between restraints is: $2 p$ $n(p, D) c \Delta D$ molecules.

In contrast to the depletion of the p population, cross-linking of longer chains of length $(p+l)$ with other nearby chains has the potential to generate new segments of length $p$. This increase in the population of chains with length $p$ can be written as:

$2 c \Delta D \sum_{l=p+1}^{p_{\max }} \frac{l n(l)}{N}\left[\sum_{k=1}^{P_{\max }} A(p, l, k) k n(k, D)\right]$

The factor of 2 in front of the above expression originates from the fact that the $(p+l)$ chains may not only develop active centers and cross-link with nearby molecules but nearby molecules may also develop active centers and cross-link with $(p+l)$ polymerized molecules. The end results in the two cases are indistinguishable.

In the above expression:

$$
\begin{aligned}
& A(p, l, k)=\frac{2}{(p+l)} \text { or } k<p \quad \mathrm{f} \\
& A(p, l, k)=\frac{2}{p+l}+\frac{2}{k}=\frac{2(p+l+k)}{(p+l) k} \quad \text { for } k \geq p
\end{aligned}
$$

The term $A(p, l, k)$ is a unitless constant that takes into account the fact that just simply because a chain of length $(p+l)$ cross-links with another chain, a length $p$ chain is not necessarily produced. Indeed, if a $(p+l)$-length chain cross-links with a $k$-length chain $(k<p)$, there are only 2 possible cross-link locations (among a total of $(p+l)$ possibilities) that would produce a chain of length $p$. In the schematic presented in Fig. 3, only a cross-link event at either location A or B would result in the formation of a $p=3$ length chain from one $(p+l)=4$ and one $k=2$ chain . In Fig. 3, each square box represents a monomer in the polymer chain. For example, a cross-linking event at location A along the $(p+l)$ chain yields a $p=3$ molecule. This result is 
illustrated in the bottom left corner of Fig. 3. A cross-linking event that does not involve either location A or B does not yield any $p=3$ chain and is illustrated in the bottom right corner of Fig. 3. When $k \geq p$, there are two additional locations (labeled C and D in Fig. 4) on the $k$-length chain which can participate in the formation of a chain with $p=3$. The possibility to form a $p=$ 3 chain in Fig. 4 is 22 over a total of 28 possibilities.

In general, cross-linking can be described mathematically by:

$$
\Delta n(p, D)=-2 p n(p, D) c \Delta D+2 c \Delta D \sum_{l=1}^{p_{\max }-p} \frac{(p+l) n(p+l)}{N}\left[\sum_{k=1}^{P_{\max }} A(p, l, k) k n(k, D)\right]
$$

Defining $c \Delta D=\Delta t$ :

$$
\Delta n(p, t)=-2 p n(p, t) \Delta t+2 \Delta t \sum_{l=1}^{p_{\max }-p} \frac{(p+l) n(p+l)}{N}\left[\sum_{k=1}^{P_{\max }} A(p, l, k) k n(k, t)\right]
$$

And by defining $n(p, t) / N=\mathrm{m}(p, t)$, the rate equation for the population evolution of an arbitrary length $p$ becomes:

$$
\frac{\Delta m(p, t)}{\Delta t}=-2 p m(p, t)+2 \sum_{l=1}^{p_{\max }-p}(p+l) m(P+l, t)\left[\sum_{k=1}^{P_{\max }} A(p, l, k) k m(k, t)\right]
$$

\section{Chain-Scissioning:}

For chain-scissioning, $s$ is defined as the probability that a monomer ruptures upon being irradiated. The decrease in the $p$ population due to a rupture within that population for an incremental dose $\Delta D$ is then: $p n(p, D) s \Delta D$ molecules. However, a scission event in a longer chain $(l>p)$ may result in the formation of a $p$-length chain. The total increase in the population

of chains with length $p$ due scission of longer molecular chains is: $\sum_{l=p+1}^{p_{\max }} \frac{2}{l} l n(l, D) s \Delta D$ molecules. 
The factor $2 / l$ takes into account the fact that only two scission locations on the $l$-length chain would result in the formation of a $p$-length chain. Putting things together for the case of chainscissioning: $\Delta n(p, D)=-p n(p, D) s \Delta D+\sum_{l=p+1}^{p_{\max }} \frac{2}{l} \ln (l, D) s \Delta D$

Defining $s \Delta D$ as $\Delta \tau$ yields:

$\Delta n(p, \tau)=-p n(p, \tau) \Delta \tau+\sum_{l=p+1}^{p_{\max }} \frac{2}{l} \ln n(l, \tau) \Delta \tau$

And replacing $n(p, \tau) / N=m(p, \tau)$, the rate equation for the population $p$ due to chain-scissioning becomes:

$$
\frac{\Delta m(p, \tau)}{\Delta \tau}=-p m(p, \tau)+\sum_{l=p+1}^{p_{\max }} 2 m(l, \tau)
$$

Eqns. (3) and (4) are written to be applied directly to the MQ-NMR data presented above, which in reality is discrete, not continuous as depicted for simplicity in Fig 1(b). In the case of a continuous data source, the summation would of course become integration. Note that Eqn.(4) is identical with the mathematical formula governing chain-scissioning in free polymer chains derived by Saito [17].

\section{H-LINKING, Y-LINKING, AND END-LINKING AMONG POLYMER CHAINS IN A}

\section{SILICONE NETWORK:}

\section{H-linking:}

The statistical description of the general cross-linking process described in Eqn. (3) can be applied directly with no modifications to the case of H-linking processes. Recall that in Eqn. (3), the only adjustable variable is the rate of $\mathrm{H}$-linking, $c$, in the expression $\Delta t=c \Delta D$. Some 
examples of H-linking and Y-linking are given in Fig. 5 [31]. It is important to note that because bond energies of $\mathrm{C}-\mathrm{H}$ and $\mathrm{Si}-\mathrm{C}$ bonds (roughly $4.3 \mathrm{eV}$ ) are less than the bond energy for the Si-O bond (ca. 5.3-5.9 eV), one would expect the generation of an active center to be preferable to main-chain scission; but because the differences in these bond energies are orders of magnitude smaller than the energy of the ${ }^{60} \mathrm{Co}$-emitted gamma ray $(1.17 \mathrm{MeV})$, simultaneous action of $\mathrm{H}$ linking, Y-linking, and end-linking must be considered.

\section{Y-linking:}

Y-linking is defined as the attachment of the dangling end of a chain, after a chainscissioning event, to a monomer on a nearby chain, which results in the formation of a characteristic Y-shape. In this report, Y-linking is modeled by first performing chain-scissioning as described in Eqn. (4) up to the total irradiation dose. For an arbitrary $p$ value, the first term in Eqn. (4) corresponds to the number of chains that are of $p$-length before the scission event, and the second term accounts for the number of $p$-length dangling bonds created after the scission event. These dangling bonds are taken to be distributed randomly and are subjected to a random attachment (Y-linking) to a neighboring chain with a probability of $Y$ events per monomer. Such a Y-linking process is performed sequentially after each chain-scissioning step and is expressed as:

$$
\frac{\Delta m_{Y}(p)}{Y}=-p\{m(p)-p m(p) \Delta \tau\}+\sum_{l=p+1}^{p_{\max }} 2\{m(l)-l m(l) \Delta \tau\}
$$

Here, $\Delta m_{Y}(p)$ is the change in a given $p$ population after the Y-linking process. The first and second terms in Eqn.(5) represent the action of Y-linking of dangling ends having chain lengths 
of $p$ and $(p+l)$, correspondingly. The value of $Y$ is such that the amount of new chains created through the Y-linking process is twice the amount of dangling bonds created in the preceding irradiation-induced chain scissioning process. $Y$ can be expressed mathematically in Eqn. (6):

$$
Y=\frac{2 \sum_{p=1}^{P_{l}} \sum_{l=p+1}^{P_{\max }}(2 m(l, \tau) \Delta \tau)}{\sum_{p}\left[-p\{m(p)-p m(p) \Delta \tau\}+\sum_{l=p+1}^{P_{\max }} 2\{m(l)-l m(l) \Delta \tau\}\right]}
$$

Note that for the Y-linking process, the only adjustable variable is the rate of chain-scissioning,

$s$, in the expression $\Delta \tau=s \Delta D$.

\section{End-linking:}

End-linking is the attachment of two scissioned chains at the termini of their respective dangling ends. As with Y-linking, end-linking is modeled by first performing chain-scissioning up to the total irradiation dose. The first term in Eqn. (4) is responsible for the reduction in the original number of chains of length $p$, while the second term corresponds to the dangling bond creation just as in the case of Y-linking. In the second step, random dangling ends are taken to attach to each other (end-to-end) with $100 \%$ success rate. The effect of this process on the MWBC distribution can be expressed mathematically by:

$$
\Delta m_{n e t}(p+k)=\left\{\sum_{l=k+1}^{p_{\max }} 2 m(l, \tau) \Delta \tau\right\} \frac{\sum_{l=p+1}^{p_{\max }} 2 m(l, \tau) \Delta \tau}{2 \sum_{p} \sum_{l=p+1}^{p_{\max }} 2 m(l, \tau) \Delta \tau}
$$

Here, $\Delta m_{n e t}$ is the change in the $(p+k)$-polymerized population after the end-linking process between two dangling ends, one of length $p$ and another of length $k$. Note that the end-linking 
process joins two chains at their respective dangling ends into one chain, and the factor of 2 in the denominator corrects for double-counting. Similar to the case of H-linking or Y-linking, the only adjustable variable in the end-linking process is the rate of chain-scissioning, $s$, in the expression $\Delta \tau=s \Delta D$.

\section{APPLICATION OF H-LINKING, Y-LINKING, AND END-LINKING ON MQ-NMR}

\section{DATA OF UNIRRADIATED TR55}

As seen in Fig. 1, the NMR-derived chain length distribution for the unirradiated sample consists of two well separated peaks with the smaller peak being below $p=30$. This bimodal distribution is also clearly observed for the irradiated samples. Recall that for the TR-55 materials, fumed silica is used as a reinforcing filler. Previous work on these engineering elastomers generally attributes this secondary peak of high RDC values (i.e., small $p$ values) to chains adsorbed via hydrogen bonding events to the surface of the silica. It is taken, then, that the larger and smaller peaks correspond to chains resulting from polymer-polymer events and polymer-filler events, respectively. Polymer segments associated chemically or physically with silica surfaces are much shorter (dynamically speaking, as revealed through NMR) than strands produced, broadly speaking, by polymer-polymer events. These polymer-polymer and polymerfiller events will be referred to henceforth as "interchain events" and "surface events", respectively.

Since segmental dynamics of surface-associated polymer chains are expected to be dominated by adsorption events (which can be modeled as effective cross-links), the effects of entanglements and chemical cross-links, which otherwise strongly influence the behavior of bulk 
polymer chains (i.e., interchain events), will be ignored. Because the NMR-derived MW distributions feature two distinct peaks, the evolution of interchain and surface events (as represented in Fig. (1) will be modeled independently. Refer to Fig. 6 for (a) the MW distributions of polymer chain lengths $p<30$ and (b) long chain polymers associated with interchain events. Note that since $\sum_{p} m(p, D) p=1$, the area under each curve in a complete plot of $m p$ vs. $p$ is unity and is dimensionless. Because each peak is treated separately in the subsequent analysis, the surface and interchain peaks are normalized to unity separately (as done for Fig. 6).

\section{Modeling the evolution of the MWBC distribution: Interchain Events}

For many siloxane-based elastomers, it has been reported that a typical fraction of dangling chain ends is only $\sim 2-3 \%$, and that this population typically decreases with increasing doses when irradiated in a vacuum environment $[10,26,31]$. The implication of this observation is two-fold: (1) the small fraction of dangling ends is, within experimental errors, negligible in the MW distributions obtained from MQ-NMR (though steps are taken to remove their effect on the observed MQ build-ups); and more importantly (2) chain-scissioning is largely transient and ultimately yields Y-linking, end-linking, or a combination of the two. All dangling chains formed by chain-scissioning are therefore forced to participate in subsequent $\mathrm{Y}$-linking and/or end-linking interchain events.

In Fig. 7, the MQ-derived molecular weight distributions at $10 \mathrm{kGy}, 50 \mathrm{kGy}, 100 \mathrm{kGy}$, and $250 \mathrm{kGy}$ for the interchain events are replotted in red from Fig 6(b). PB models with Hlinking, with Y-linking, and with end-linking are represented by dark long dashed-lines, dark 
short dashed-lines, and dark solid lines, respectively. The MW evolution of irradiated TR55 as measured from MQ-NMR (such as a shift to lower $p$ values and a narrowing of the distribution) can be qualitatively reproduced by the distributions predicted through the application of $\mathrm{H}$ linking alone or Y-linking alone. The best agreement between the NMR data and the PB modeling considering only H-linking alone is obtained when the rate of active center generation, $c$, is set to $2.34 \times 10^{-4}$ events per kGy per monomer. This derived value, though determined uniquely at each cumulative dose, remains roughly constant for all doses considered. However, the quality of the agreement between the modeled and the experimental distributions worsens at higher doses. In addition, modeling with H-linking alone tends to grossly over-predict the population distribution at the low $p$ values (note the discrepancies between the modeled and NMR distributions at $p<50$ ). Similar behavior is also observed when applying Y-linking alone. Indeed, except for slight differences at $100 \mathrm{kGy}$ (see Fig. 7(c)), the PB predicted curves for Ylinking have the best agreement with MQ-NMR data and are almost indistinguishable from those for $\mathrm{H}$-linking when the rate of chain-scissioning, $s$, is set to $2 c / 3$. Since $2 / 3$ is the theoretical ratio of new chains formed by H-linking to those formed by Y-linking, this suggests the inability of the PB modeling presented in this work to distinguish unambiguously between H-linking and Y-linking.

In contrast, the PB-based predicted curve for end-linking alone at $10 \mathrm{kGy}$ has an almost perfect fit with MQ-NMR data when the rate of chain-scissioning, $s$, is set to be $5 c / 3$. Unlike the case of H-linking alone and Y-linking alone, the PB-based end-linking modeling does not overpredict the population distribution at lower degrees of polymerization. However, end- 
linking predictions tend to bias MWBC distributions toward larger molecular weight product chains. The residual sum of squares for the end-linking modeling at $10 \mathrm{kGy}$ is much better than those for $\mathrm{H}$-linking and $\mathrm{Y}$-linking; but above $50 \mathrm{kGy}$, the residual sum of squares for end-linking becomes much larger than those for H-linking and Y-linking. Note that in random end-linking, two dangling chains bond to form longer chains, hence end-linking tends to preserve the original MWBC distribution at lower doses and to shift the distribution toward large $p$ values at higher doses. Clearly, end-linking alone cannot be responsible for the change in the molecular weight distribution observed by MQ-NMR.

In consideration of the limited accuracy of individual linking models to reproduce the observed MW distributions, it becomes more logical to employ a mixed-linking model that covers the physics/chemistry of not just $\mathrm{H}$-linking (or equivalently Y-linking at a rate of $2 c / 3$ ), but also of end-linking. Simultaneous H-linking, Y-linking, and end-linking among the polymers chains are realized numerically by performing each type of linking sequentially (in any order). However, due to the approximate nature of the equations governing $\mathrm{H}$-linking, $\mathrm{Y}$ linking, and end-linking derived above and the truncation errors inherently associated in numerical calculations, after each round of numerical iteration ( $\Delta D=1 \mathrm{kGy})$, the total number of monomers should be carefully normalized to that for the unirradiated sample such that there is no gain or loss in the total amount of monomers. The more physics/chemistry a model covers, the better fit with experimental data is expected, but at the cost of increasing the number of often arbitrarily adjustable variables. As discussed in the previous section, the best agreements with MQ-NMR data are obtained when the H-linking alone, Y-linking alone, and end-linking alone 
have the rates of $c, 2 c / 3$, and $5 c / 3$, respectively. In the absence of any further knowledge on the actual participation rates of the three types of linking and if an equal weighting approach is chosen, this ratio of $c: 2 c / 3: 5 c / 3$ can reasonably serve as a constraint and preserves a fitting algorithm that requires only a single adjustable parameter. In Fig. 8, a mixed-linking model with a rate of $c=7.8 \times 10^{-5}$ events per kGy per monomer for H-linking while having a constraining ratio of $c: 2 c / 3: 5 c / 3$ for the corresponding $\mathrm{H}$-linking, $\mathrm{Y}$-linking, and end-linking processes are presented and compared with MQ-NMR data. The level of over-prediction for the distribution at lower degrees of polymerization is observably smaller in the case of mixed-linking modeling than in the cases of just H-linking or Y-linking alone. The fit with MQ-NMR data at higher irradiation doses also improves at higher irradiation doses (at $100 \mathrm{kGy}$ and $250 \mathrm{kGy}$ ) with the mixed-linking modeling.

\section{Surface Events:}

For polymer chains adsorbed to silica surface, the chain segments in between any two adsorption sites are expected to be short and that they mostly cross-link to the surface at active centers created by radiation exposure. As discussed above, this surface association is a logical cause for the smaller peaks in observed molecular weight distributions presented in Fig. 1 and Fig. 6(a). None of the equations developed for H-linking, Y-linking, or end-linking, however, proves adequate to model the evolution of the MW distribution. This fact is unsurprising, as the mathematical expressions for surface cross-linking are different than those developed for interchain events considered above. The mathematical expression that describes such a surface event is written as: 


$$
\frac{\Delta m(p, T)}{\Delta T}=-2 p m(p, T)+\sum_{l=p+1}^{p_{\max }} 4 m(l, T)
$$

A decrease in the population of chains with length $p$ occurs when a chain in that population develops an active center and cross-links with the silica surface or when an active center on the silica surface, which has an abundance of SiOH bonds [32], cross-links with a polymer strand of length $p$. This is responsible for the first term on the right-hand side of Eqn. (8) which is identical with the first term in Eqn. (3) for the case of H-linking (provided that one of the two polymer chains involved in the H-linking is replaced with the silica surface). The second term on the right-hand side of Eqn. (8) is the increase in the population of polymer of length $p$ due to the development of an active center along the strand length of some $(p+l)$ polymer strands and subsequent reaction of this chain to the silica surface. This is analogous to the chain-scissioning of $(p+l)$ polymers described in the second-term on the right-hand side of Eqn. (4). However, even when there is no active center along the strand length of a $(p+l)$ polymer strand, this $(p+l)$ polymer strand can still react with active centers developed on the silica surface to form a $p$ polymer. For this reason, the second term on the right-hand side of Eqn. (8) is twice the second term on the right-hand side of Eqn. (4) which considers chain-scissioning. In term of mathematical expression, Eqn. (8) is similar to Eqn. (4), except that $\Delta \tau$ in Eqn. (4) is replaced by $\Delta T=g \Delta D$ in Eqn. (8). Here $g$ is the effective rate at which cross-linking occurs between the short polymer segments and the silica surface. 
Unfortunately, even Eqn.(8) fails to qualitatively describe the trend in the evolution of the MWBC distribution of the smaller peak presented in Fig.6(a). However, if $\Delta T$ in Eqn. (8) is replaced by $p \Delta T$ as seen in Eqn. (9) below, the evolution trend of the short polymer strands on silica surfaces can be approximated.

$$
\Delta m(p, T))=-2 p m(p, T)\{p \Delta T\}+\sum_{l=p+1}^{p_{\max }} 4 m(l, t)\{l \Delta T\}
$$

Note that in Eqn. (9), $g$ is now modified to have the unit of events per $1 \mathrm{kGy}$ per monomer squared instead of events per $1 \mathrm{kGy}$ per monomer as in Eqn. (8). This squared dependence suggests that a cross-linking event depends on the probabilities of active center formation on both a chain and the surface. From a chemistry perspective, the requirement of simultaneous active center generation is unexpected. A more likely explanation involves the reduced segmental fluctuations of chains adsorbed to the silica surface. It is supposed then that polymers immobilized through hydrogen bonding interactions with the surface making them glassier vis-àvis their melt state counterparts. This restricted mobility would then be expected to significantly increase the chance of the recombination of a scissioned chain to its original configuration. Accounting for this possibility in a future model might help better understand the physical origin of the parameter. In Fig. 9, a silica-polymer interaction model with a rate of $g=5.07 \times 10^{-4}$ events per $1 \mathrm{kGy}$ per monomer square are presented and compared with MQ-NMR data at 10 kGy (a), 50 kGy (b), 100 kGy (c), and 250 kGy (d). From Fig. 9, it is observed that statistical modeling can predict the trend of the silica-polymer events with increasing irradiation levels. 
But similar to the case of interchain events, the presented modeling for the cross-linking of polymer to silica surfaces also tends to lose it quantitative agreement with MQ-NMR at higher irradiation doses (250 kGy in this report).

\section{CONCLUSION:}

The statistical methodology of population balance has been employed to model the evolution of the MWBC distribution as a function of irradiation for TR55 silicone. The fits of MQ-NMR data by predictive modeling presented in this manuscript are only qualitatively similar, but allow for the establishment of relative bounds on rates of the competing radiation induced mechanisms. Further, this is the first time that MW distributions for cross-linked siloxane-based elastomers obtained from MQ-NMR have been analyzed using the methodology of population balance. The data obtained herein suggest the co-existence of $\mathrm{H}$-linking, $\mathrm{Y}$ linking, and end-linking. The ability of this methodology to distinguish between H-type and Ytype crosslinking, so far is minimal. Perhaps with improved fitting algorithms for the MQ-NMR growth curves and improved experimental protocols for oversampling early times in the growth curve, improved analysis will be possible. However, the method clearly demonstrates the ability to detect differences in degradation due to differences in network structure motifs. The general approach described in this report to arrive at the rate equations for chain-scisioning and crosslinking can be extended to other siloxane-based elastomers for the kinetic prediction of the evolution of the molecular weight distribution under irradiative, thermal, or mechanical aging.

Further, the method described here is general to MWBC distributions obtained from any 
experimental or theoretical methodology and should prove to be quite valuable for the characterization of network changes in elastomers.

\section{REFERENCES:}

1. G. Kraus, Rubber Chem. Tech. 38, 1070 (1965).

2. J. M. Zeigher, F. W. G. Fearon, Silicon Based Polymer Science: A Comprehensive Resource, Vol. 224, ACS Press (1990).

3. P. Vondracek, A. Pouchelon, Rubber Chem. Technol. 63, 202 (1990).

4. A. Charlesby, Proc. R. Soc. Lond. A230(1180), 120 (1955).

5. A. A. Miller, J. Am. Chem. Soc. 82, 3519 (1960).

6. A. S. Palsule, S. J. Clarson, C. W. Widenhouse, J. Inorg. Organomet. Polym. 18, 207 (2008).

7. E. L. Warrick, Ind. Eng. Chem. 24, 842 (1955).

8. A. A. Basfar, Radiat. Phys. Chem. 50, 607 (1997).

9. J. E. Mark, D. W. McCarthy, Rubber Chem. Technol. 71, 941 (1998).

10. A. Chien, R. Maxwell, D. Chambers, B. Balazs, J. LeMay, Radiat. Phys. Chem. 59, 493 (2000).

11. R. Maxwell, R. Cohenour, W. Sung, D. Solyom, M. Patel, Polym. Degrad. Stab. 80, 443 (2003).

12. W. Burlant, V. Serment, J. Neerman, J. Polym. Sci. 58:491 (1962).

13. J. E. Mark, ed. Physical properties of polymers handbook (chapter 41: Effects of Electron Bean and g-Irradiation on Polymeric Materials by K. Dawes, L. C. Glover), AIP press, Woodbury, NY, (1996). 
14. C. G. Delides, I. W. Sheppard, Radiat. Phys. Chem. 10, 379 (1977).

15. M. Celina, K. T. Gillen, R. A. Assink, Poly. Deg. Stab. 90, 395 (2005).

16. A. Charlesby, S. H. Pinner, Proc. R. Soc. London A 249, 367 (1959).

17. O. Saito, J. Phys. Soc. Jpn. 13, 198 (1958).

18. R. H. Boyd, J. Chem. Phys. 31, 321 (1959).

19. R. H. Boyd, T. -P Lin, J. Chem. Phys. 45, 773 (1966).

20. W. Chasse, J. L. Valentin, G. D. Genesky, C. Cohen and K. Saalwachter, J. Chem . Phys. 134 (4) (2011).

21. K. Saalwachter, P. Zie gler, O. Spyckere lle, H. Haider, A. Vidal and J.-U. So mmer, J. Chem. Phys. 119, 3468-3482 (2003).

22. K. Saalwächter, J. Am. Chem. Soc. 125, 14684 (2003).

23. R. S. Maxwell, S. C. Chinn, C. T. Alviso, C. A. Harvey, J. R. Juliani, T. S. Wilson, R. Cohenour, Polym. Degrad. Stab. 94, 456 (2009).

24. K. Saalwächter et al., J. Chem. Phys. 119, 3468 (2003).

25. J. R. Giuliani, E. L. Gjersing, S. C. Chinn, T. V. Jones, T. S. W ilson, C. T. Alviso, J. L. Herberg, M. A. Pearson, and R. S. Maxwell, J. Phys. Chem. B 111, 12977 (2007).

26. R. S. Maxwell, S. C. Chinn, C. T. Alviso, T. S. Wilson, J. Herberg, R. Cohenour, Polym. Deg. Stab. 94, 456 (2009).

27. S. C. Chinn, C. T. Alviso, E. S. F. Berman, C. A. Harvey, R. S. Maxwell, T. S. Wilson, R. Cohenour, K. Saalwächter, W. Chassé, J. Phys. Chem. B, 114, 9729 (2010).

28. P. Sotta and B. Deloche, Macromolecules 23 (7), 1999-2007 (1990).

29. N. Neuburger, I. Bahar and W. L. Mattice, Macromolecules 25 (9), 2447-2454 (1992).

30. K. Saalwachter, B. Herrero and M. Lopez-Manchado, Macrom olecules 38 (23), 96509660 (2005).

31. S. P. Aniruddha et al., J. Inorg. Organomet. Polym. 18, 207 (2008). 
32. L. N. Dinh, A. K. Burnham, M. A. Schildbach, R. A. S mith, R. S. Maxwell, B. Balazs, W. McLean II, J. Vac. Sci. Technol. A 25, 597 (2007).

\section{FIGURE CAPTIONS:}

Fig. 1: The relative probability distributions obtained from MQ-NMR data for TR55 exposed to $10 \mathrm{kGy}, 50 \mathrm{kGy}, 100 \mathrm{kGy}$, and $250 \mathrm{kGy}$ in a semi-log plot (a) and in a linear plot (b).

Fig. 2: Cartoons depicting active centers and chain-scissioning form ed under gamma irradiation.

Fig 3: For the case involving 2 polymers, one with a chain length of $p+l=4$ and one with a chain length of $k=2$, only a cross-link at either location A or location B on the $(p+l)$ chain would result in the formation of a $p=3$ length chain.

Fig. 4: For the case involving 2 polym ers, one with a chain length of $p+l=4$ and one with a chain length of $k=7$, cross-linking at locations $\mathrm{A}$ or $\mathrm{B}$ on the $(p+l)$ chain or at locations $\mathrm{C}$ or $\mathrm{D}$ on the $k$ chain yields molecules with $p=3$. The possibility to form a $p=3$ molecule in Fig. 4 is 22 over a total of 28 possibilities.

Fig. 5: A schematic of possible structures formed via H-linking and Y-linking.

Fig. 6: The distributions associated with surface events responsible for polymer populations having chains with $p<30$ are presented in (a) while those of interchain events responsible for 
polymer populations having much longer chains are shown in (b).

Fig. 7: The molecular weight distributions at $10 \mathrm{kGy}, 50 \mathrm{kGy}, 100 \mathrm{kGy}$, and $250 \mathrm{kGy}$ for the interchain events (see Fig. 6(b)) obtained from MQ-NMR, PB m odeling with H-linking, PB modeling with Y-linking, and PB modeling with end-linking are represented by the red solid lines, the dark long dashed-lines, the dark sh ort dashed-lines, and the dark solid lines, respectively.

Fig. 8: A mixed-linking model with a rate of $c=7.8 \times 10^{-5}$ events per $1 \mathrm{kGy}$ per monom er for $\mathrm{H}$-linking while having a constraining ratio of $\quad c: 2 c / 3: 5 c / 3$ for the corresponding $\mathrm{H}$ linking, Y-linking, and end-li nking processes are presented and compared with MQ-NMR data.

Fig. 9: A silica-polymer interaction model with a rate of $g=5.07 \times 10^{-4}$ event per $1 \mathrm{kGy}$ per monomer square are presented and compared with MQ-NMR data at $10 \mathrm{kGy}$ (a), $50 \mathrm{kGy}$ (b), $100 \mathrm{kGy}(\mathrm{c})$, and $250 \mathrm{kGy}(\mathrm{d})$. 


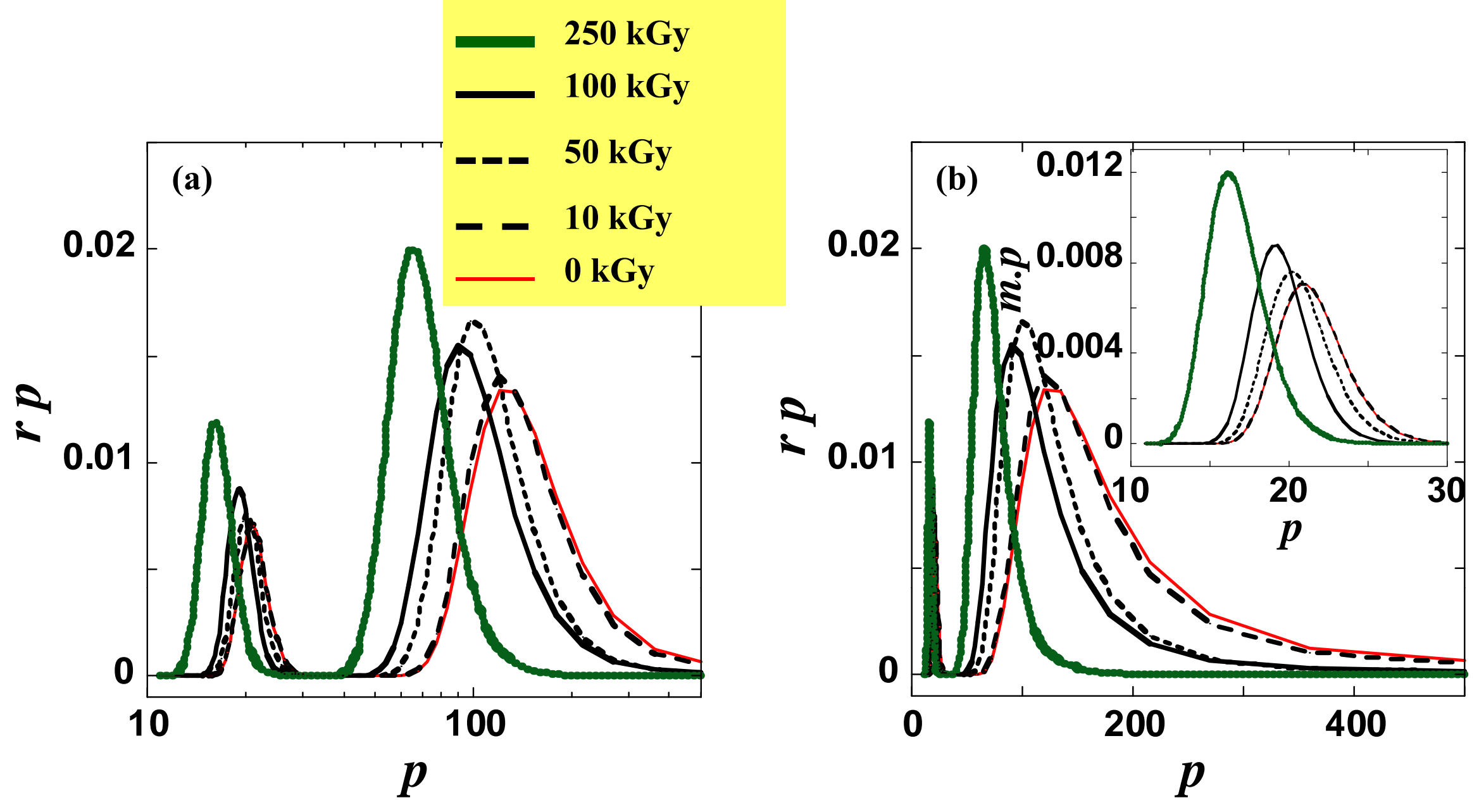

Fig. 1 


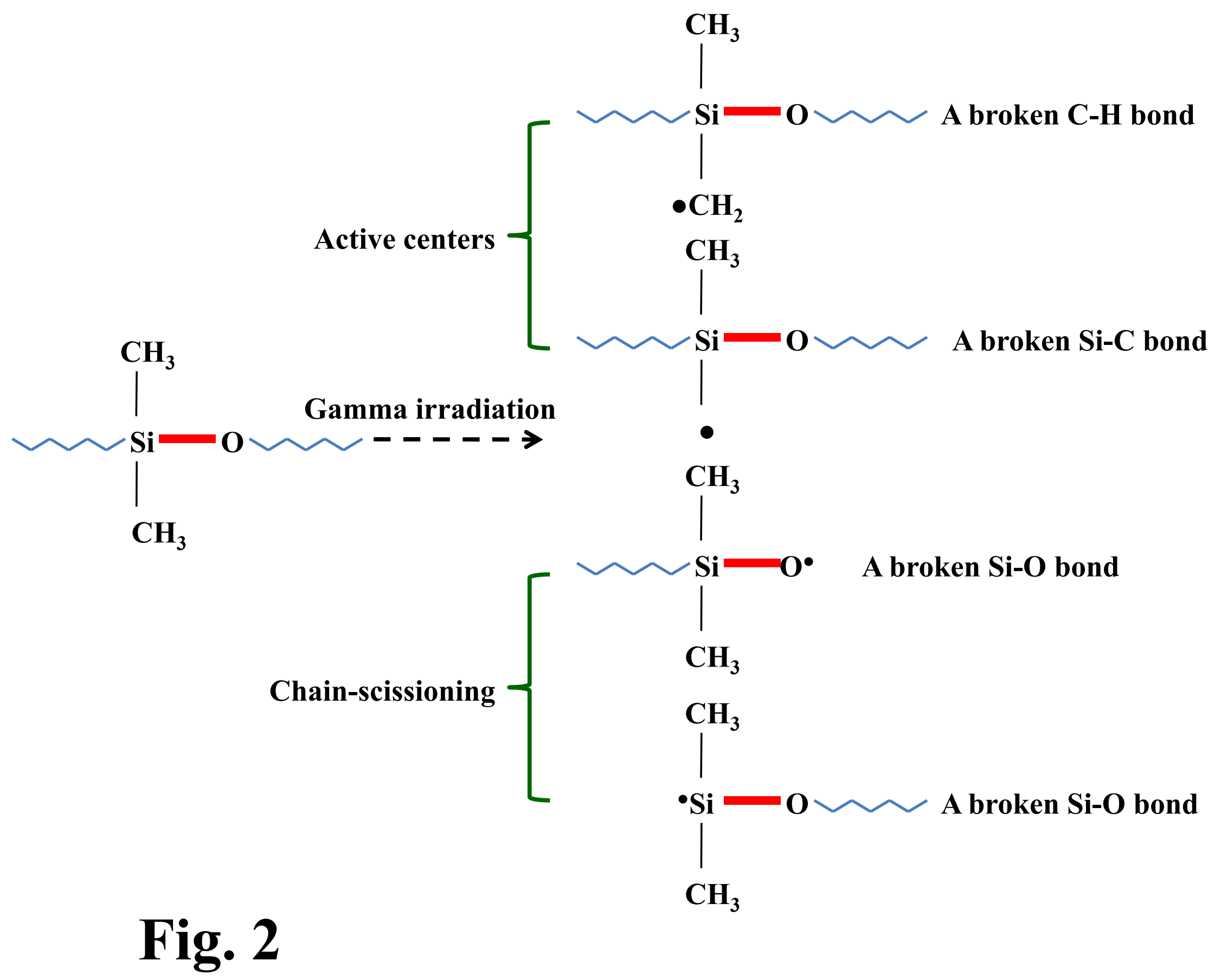




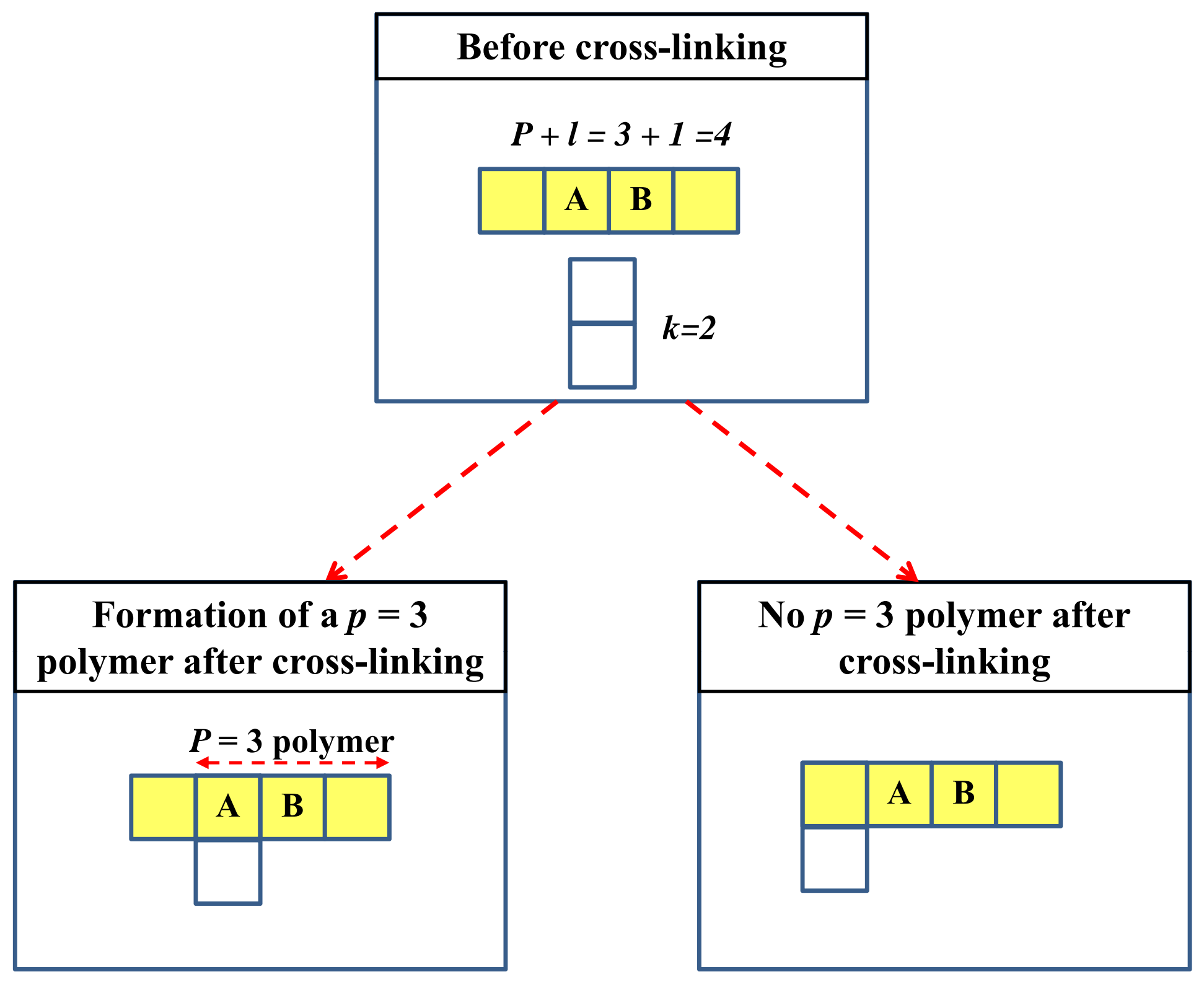

Fig. 3 


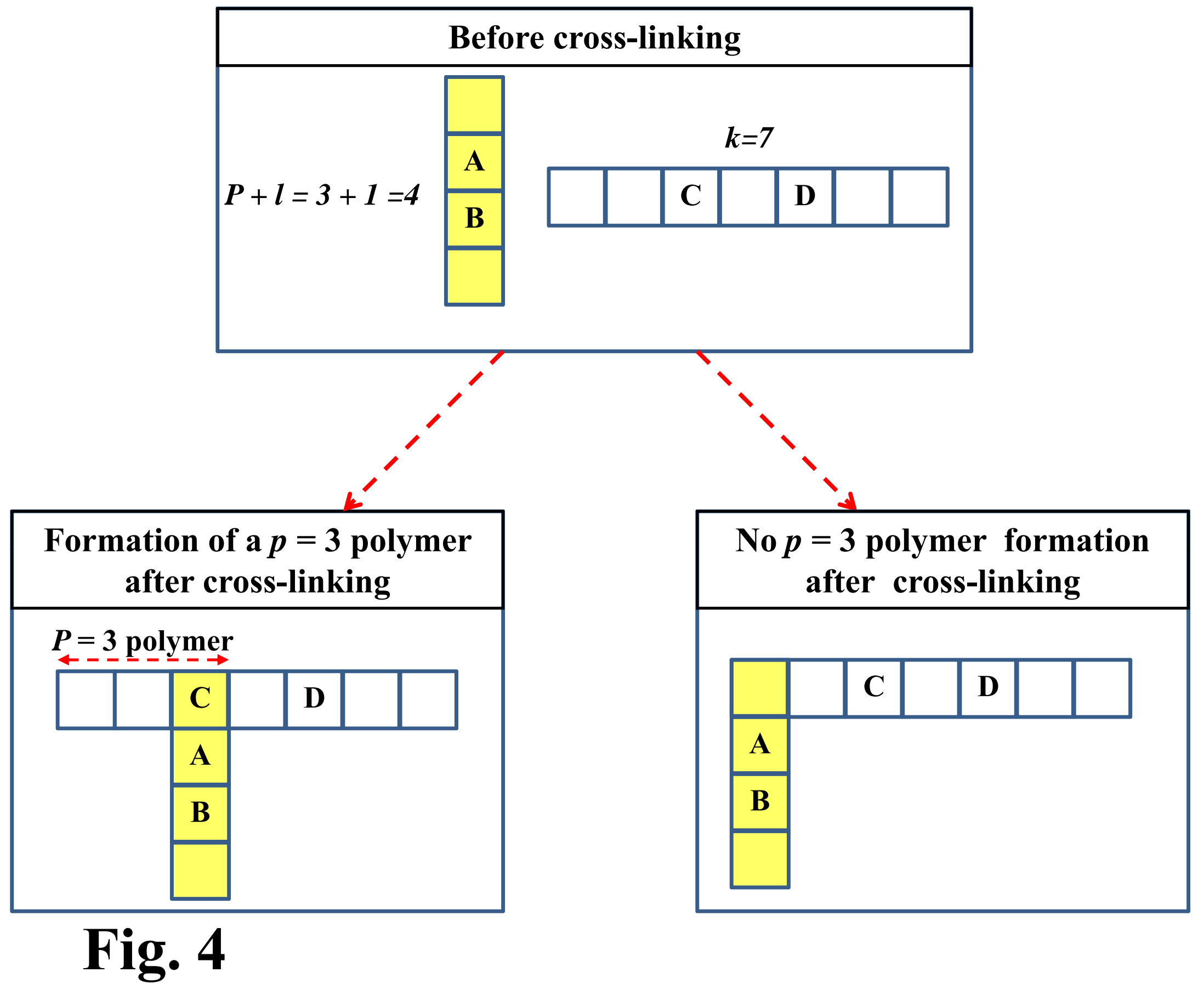


Some examples of $\mathrm{H}$-linking

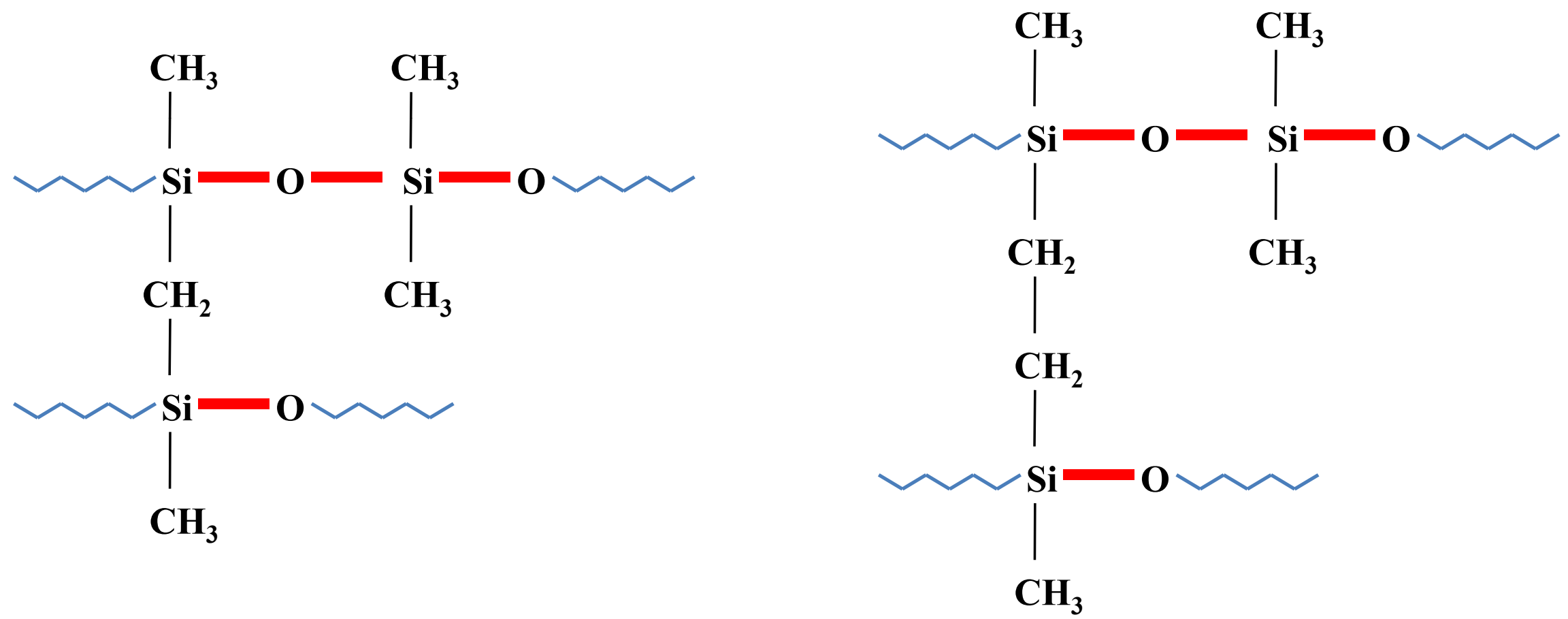

Some examples of Y-linking

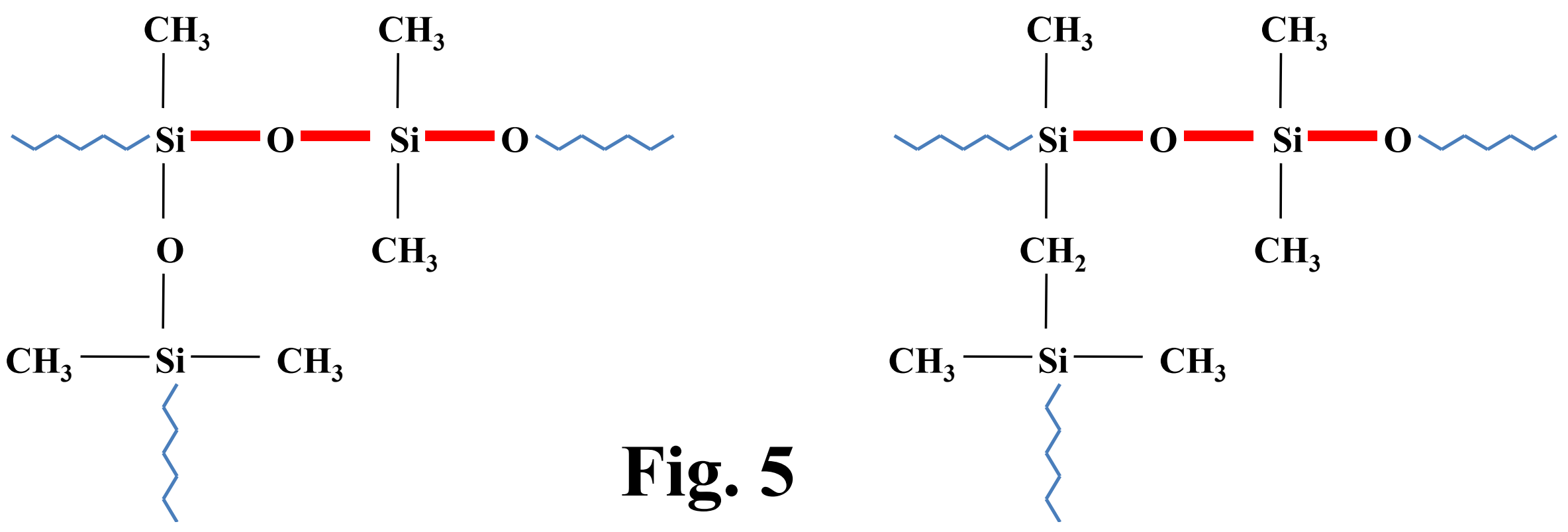



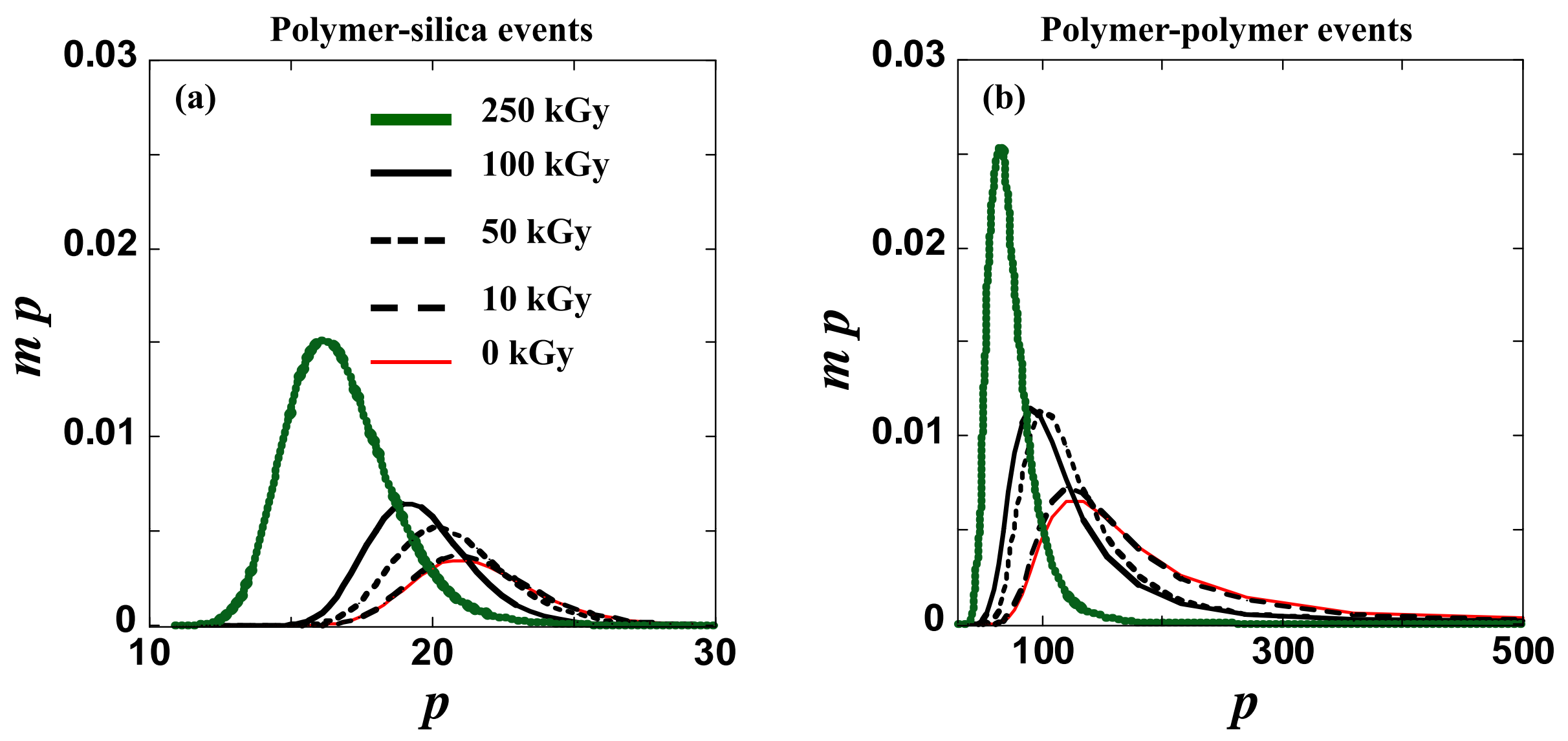

Fig. 6 

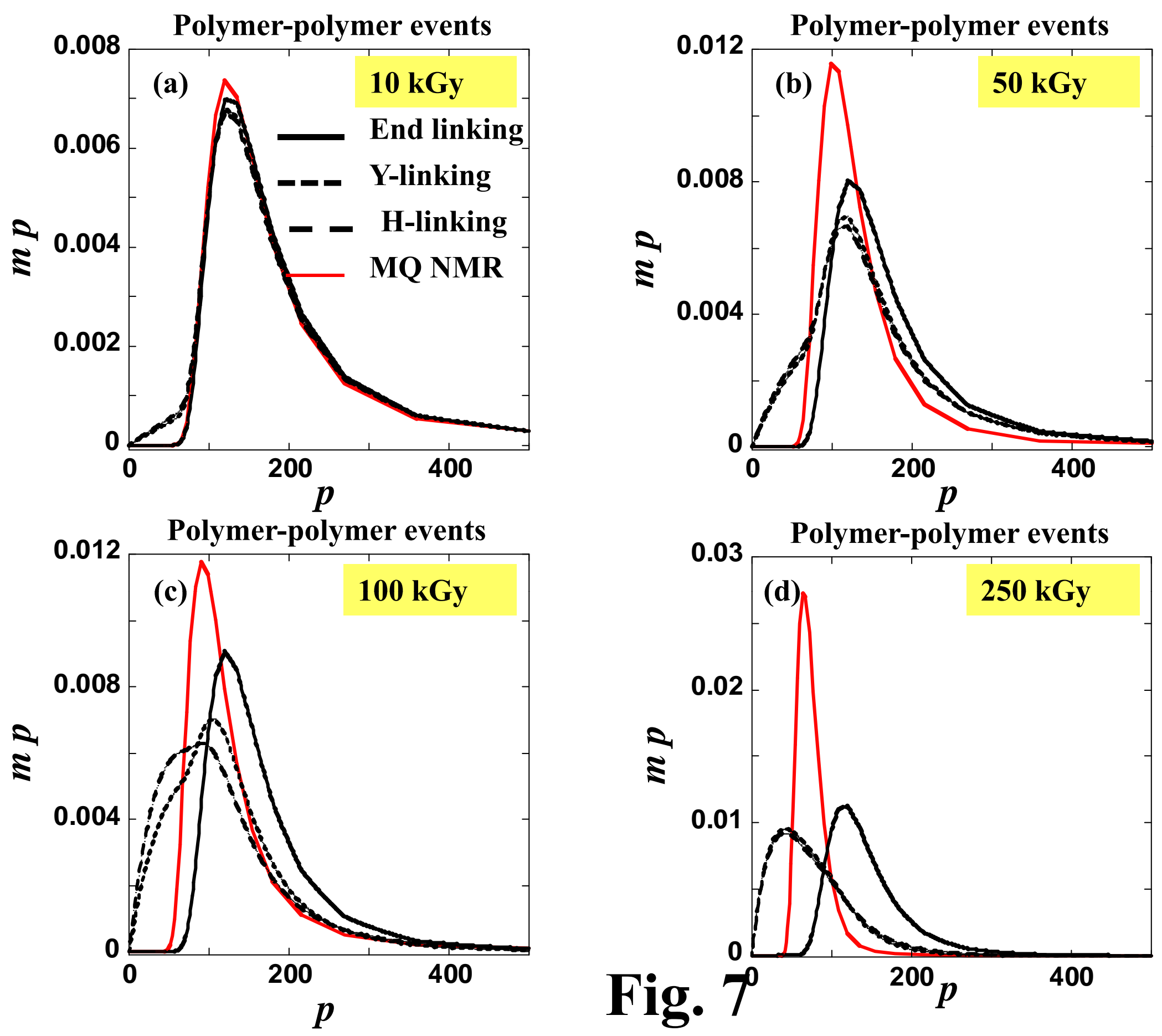

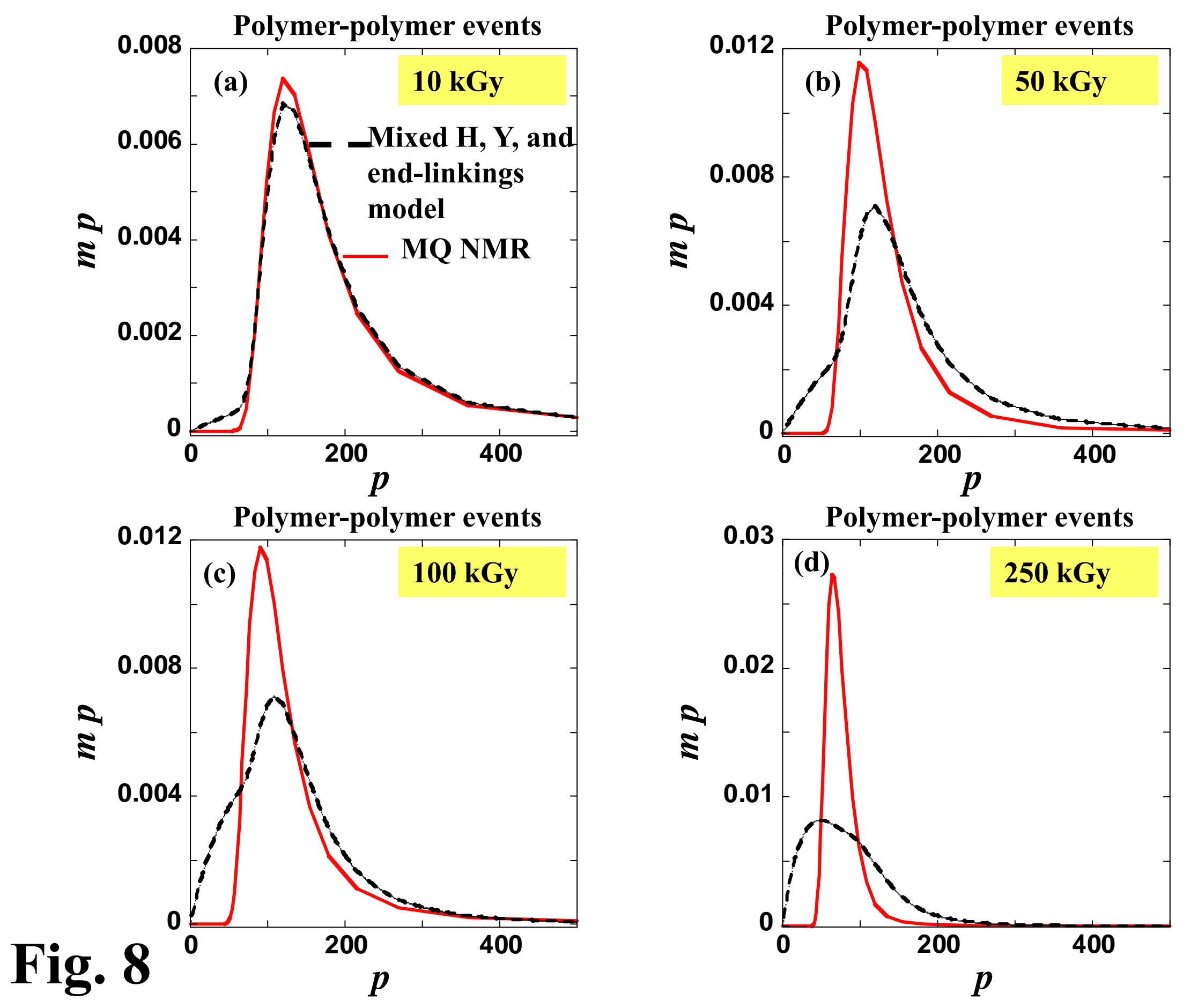

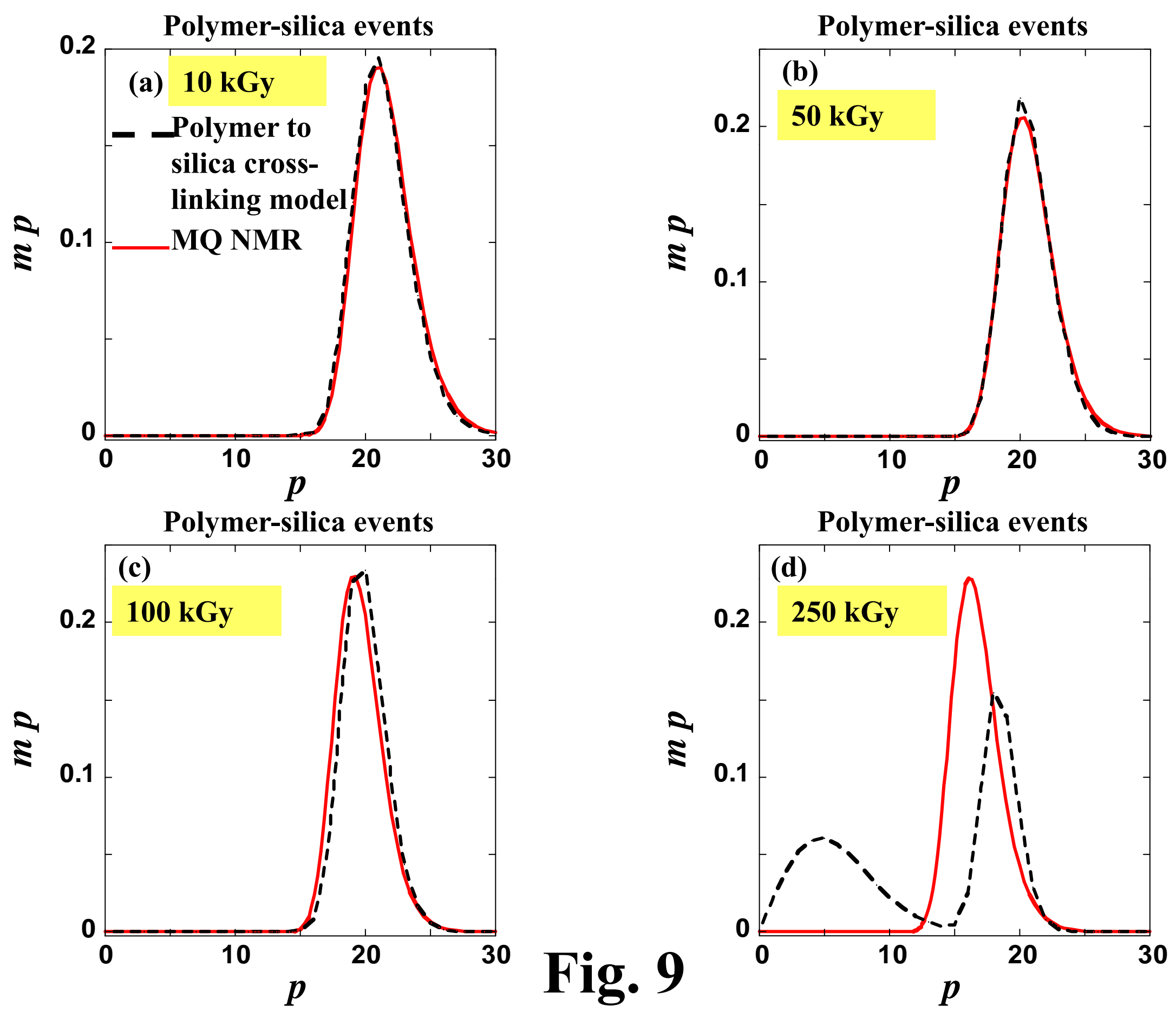